\title{
Biochemical Status of Animal Organism Under Conditions of Technogenic Agroecosystem
}

\author{
Rinat R. Fatkullin \\ Doctor of Sciences (Biology) \\ South Ural State Agrarian University \\ Troitsk, Russia \\ e-mail: dr.fatkullin@yandex.ru \\ Vladimir I. Kosilov \\ Doctor of Sciences (Agriculture) \\ Orenburg State Agrarian University \\ Orenburg, Russia
}

\author{
Evgeniya M. Ermolova \\ Doctor of Sciences (Agriculture) \\ South Ural State Agrarian University \\ Troitsk, Russia \\ Yuliya V. Matrosova \\ Doctor of Sciences (Agriculture) \\ South Ural State \\ Agrarian University \\ Troitsk, Russia
}

\author{
Svetlana A. Chulichkova \\ Candidate of Sciences (Biology) \\ South Ural State \\ Agrarian University \\ Troitsk, Russia
}

\begin{abstract}
The biochemical characteristic of the conditions of systems allows reviewing the nature and intensity of diseases (pathogenesis). To study the adaptation of the animal organism to the conditions of ecosystem pollution by toxic elements and to establish the specific metabolism routes, we have investigated a number of biochemical indicators of protein, carbohydrate, lipidic and mineral metabolism of cows. Our suggestion on lowered antioxidative function of cow blood plasma is confirmed by high concentrations of $\beta$-lipoproteins. Their level in animals is $8.00 \pm 0.11$ and $7.28 \pm 0.11 \mathrm{~g} / \mathrm{l}(\mathrm{P}<\mathbf{0 . 0 0 1})$, correspondingly, which is higher than the reference values by $20.3 \ldots 32.01 \%$. The results of biochemical studies of cow blood serum demonstrating the indicators of protein metabolism in the organism and comparison of them with reference data allow suggesting that the complex and sophisticated impact of anthropogenic environment pollution cause pseudoadaptation of animals that temporarily compensate hidden pathologic processes. In general, the established changes witness characteristic disturbance of intermediate lipide metabolism in studied cows, which is expressed as lipidemia and hyper- $\beta$-proteinemia.
\end{abstract}

Keywords-ontogenesis, biochemical characteristics, metabolism, protein fractions, environment pollution, biosynthesis.

\section{INTRODUCTION}

The periods and stages of ontogenesis and pathologic conditions are usually characterized by biochemical reactions lying in the basis of adaptation processes of animal organism or separate organs to particular environment that are supported by the following interconnected factors of biochemical adaptation: 1) genetically conditioned, 2) neuroendocrinal and 3) immunobiological [4].
The biochemical characteristic of the conditions of systems allows updating the nature and intensity of diseases (pathogenesis). To study the adaptation of the animal organism to the conditions of ecosystem pollution by toxic elements and to establish the specific metabolism routes, we have investigated a number of biochemical indicators of protein, carbohydrate, lipidic and mineral metabolism of cows. The laboratory tests were selected according to the criteria of clinical and biochemical evaluation of pathologic processes in animal organism [8].

\section{IMPLEMENTATION}

To accomplish the stated task, in Rassvet company, a group of cows has been formed as per the principle of analogues: breed is Kazakh white head, age is $6 \ldots 7$ years, live weight is $500 \ldots 520 \mathrm{~kg}$, gestation length is $3.0 \ldots 3.5$ months (I group). A similar group of cows was formed in Novokatenino company characterized by similar biogeochemical conditions (II group).

The obtained results were compared with reference values of metabolism in the organism of cattle. The concentration of total protein and protein fractions in the cow blood serum show that the organism of cows in the test groups affected by negative environmental factors demonstrate negligible decrease in total protein content and appreciable changes to the proteinogram. Moreover, the protein composition of the cow blood serum from a technogenic zone suffers substantial alteration, as compared to normal protein spectrum. The conclusions on the changes to the content of protein fractions was drawn relying on the results of statistical processing of received data [3] 
Table 1 shows that the content of total protein for cows from the 1 st group amounts to $82.23 \pm 0.32 \mathrm{~g} / \mathrm{l}$, which is lower than the normal value by $4.3 \%(\mathrm{P}>0.05)$. The animals of the 2nd group demonstrate more prominent decrease in the level of total protein, $79.31 \pm 0.42 \mathrm{~g} / \mathrm{l}$, which primarily testifies the suppression of protein biosynthesis in the organism due to impaired hepatic protein synthesis.
The analysis of the proteinogram witnesses that the decreased level of total protein mainly occurs due to lowmolecular fraction of albumins, copper-containing protein caeruloplasmin and protective proteins: $\beta_{2}$-globulins and $\gamma$ globulins, which leads to the redistribution of protein fractions in cow blood serum.

TABLE I. CONTENT OF TOTAL PROTEIN AND PROTEIN FRACTIONS IN COW BLOOD SERUM

\begin{tabular}{|c|c|c|c|c|c|c|c|c|c|c|}
\hline \multirow{3}{*}{\multicolumn{2}{|c|}{ Indicator }} & \multirow{3}{*}{ Total protein } & \multicolumn{7}{|c|}{ Serum protein } & \multirow{3}{*}{$\beta-L p$} \\
\hline & & & \multirow{2}{*}{$A l b$} & \multirow{2}{*}{ Palb } & \multirow{2}{*}{$T f$} & \multirow{2}{*}{$C p$} & \multicolumn{3}{|c|}{ Globulins } & \\
\hline & & & & & & & $\beta 2-$ & $\alpha 2-m a c r o-+\gamma 1$ & $\gamma 2-$ & \\
\hline Reference value & $\overline{\mathrm{X}} \pm \mathrm{Sx}$ & $85.92 \pm 0.21$ & $40.32 \pm 0.24$ & $7.52 \pm 0.04$ & $8.09 \pm 0.01$ & $2.42 \pm 0.04$ & $4.82 \pm 0.03$ & $7.11 \pm 0.02$ & $9.92 \pm 0.02$ & $6.06 \pm 0.07$ \\
\hline \multirow{2}{*}{ I } & $\overline{\mathrm{X}} \pm \mathrm{Sx}$ & $82.23 \pm 0.32$ & $32.85 \pm 0.18$ & $9.48 \pm 0.08$ & $10.46 \pm 0.002$ & $1.54 \pm 0.03$ & $4.31 \pm 0.03$ & $6.42 \pm 0.04$ & $8.65 \pm 0.07$ & $8.0 \pm 0.11$ \\
\hline & {$[\%]$} & -4.30 & -18.53 & +26.05 & +29.29 & -36.36 & -10.59 & -10.71 & -12.81 & +32.01 \\
\hline \multirow{2}{*}{ II } & $\overline{\mathrm{X}} \pm \mathrm{Sx}$ & $79.31 \pm 0.42$ & $31.24 \pm 0.08$ & $9.34 \pm 0.05$ & $10.84 \pm 0.03$ & $1.52 \pm 0.09$ & $4.10 \pm 0.01$ & $6.47 \pm 0.07$ & $8.01 \pm 0.11$ & $7.28 \pm 0.10$ \\
\hline & {$[\%]$} & -7.70 & $\mu-22.52$ & +24.20 & +33.99 & -37.19 & -14.94 & -9.01 & -19.25 & +20.13 \\
\hline
\end{tabular}

For instance, the concentration of serum albumin having a unique capability of transporting physiological metabolites and regulating their level in the organism due to low molecular mass, is reduced as compared to normal values in animals of the 1 st group by $18.53 \%$ and by $22.52 \%$ in animals of the 2nd group. This, firstly, indicates the suppression of anti-toxic function of albumins and appearance of separate metabolites in free biologically active state, and secondly, the reduction of albumin concentration consequently retards the renewal of the organism cellular structure proteins.

The noted situation in the proteinogram is heightened by increased concentration of post-albumins 1.26 times in the $1 \mathrm{st}$ group and 1.24 times in the 2 nd group, which can inhibit the enzymatic catalysis under intense normal or pathologic proliferative processes and having immunosuppressive activity.

The immunosuppressive activity is also featured by $\alpha_{2}$ macroglobulin- and haptoglobin-zones of $\alpha_{2}+\gamma_{1}$-globulin fraction. However, the total concentration of protein fractions of this zone turned out to be lowered for the animals from both groups by $9.01 \ldots 10.71 \%$, as compared to reference values. We believe that the low concentration of the $\alpha_{2}+\gamma_{1}$-globulin fraction is evidently conditioned by the capability of $\alpha_{2}$ macroglobulin to bind and transport nickel as nickelplasmin. In human organism, this protein contains $43 \%$ of total amount; however, the physiological function of this metalloprotein is unknown.

The content of ferroprotein fraction (Tf), conversely, increases, and its concentration in animals of the 1st group amounts to $10.46 \pm 0.02 \mathrm{~g} / \mathrm{l}(\mathrm{P}<0.001)$, in the 2 nd group $10.84 \pm 0.03 \mathrm{~g} / 1(\mathrm{P}<0.001)$, which is higher than the reference value by $31.64 \%$ on average. From our perspective, this increase is due to the peculiarities of their structure, in addition to the participation of transferrins in haemopoiesis. It was established that one of the most important components of the prosthetic group of transferrins are sialic acids that are somewhat a barrier against an infection even before the generation of specific antibodies, because transferrin nonsaturated by iron suppresses the development of viruses and bacteria. It is also widely known that sialic acids are acylated derivatives of neuraminic acid, a labile component of glycoprotein. The research results have revealed that the level of neuraminic acid in cow blood serum amounts to $41.95 \pm 2.31$ $(\mathrm{P}<0.01)$ and $45.31 \pm 1.95 \mu \mathrm{mol} / 1 \quad(\mathrm{P}<0.001)$, correspondingly to the groups of animals. Probably, the established decrease of the neuraminic acid level by $20.79 \%$ on average is connected with elevated synthesis of its derivatives. Besides, the content of transferrins in blood serum can serve as the indicator of pathologic processes in an organism. A number of authors believe that increased concentration of transferrins has positive effect.

The concentration of other transport protein of $\alpha_{2}$-globulin fraction-copper-containing oxidase (caeruloplasmin)- has declining trend both in the 1 st and 2 nd groups and amounts to $1.54 \pm 0.03(\mathrm{P}<0.001)$ and $1.52 \pm 0.09 \mathrm{~g} / 1(\mathrm{P}<0.01)$, respectively, in both groups (Table 1). Despite the abundance of biological properties of caeruloplasmin, the decrease of its level, how wee see it, is connected with the suppression of antioxidation activity of copper oxidase, which activates the autooxidation of lipides and accumulation of toxic superoxide anion radicals in cows dwelling in technogenic zones, which is a product of incomplete aerobic metabolism [10].

Our suggestion on lowered antioxidative function of cow blood plasma is confirmed by high concentrations of $\beta$ lipoproteins. Their level in animals is $8.00 \pm 0.11$ and 
$7.28 \pm 0.11 \mathrm{~g} / 1 \quad(\mathrm{P}<0.001)$, correspondingly, which is higher than the reference values by $20.3 \ldots 32.01 \%$ (Table 1 ).

The shift of the protein trend in cows' organisms appreciably altered the level of $\beta_{2}$-globulins, which are the blood protective proteins. The concentration of these proteins turned out to be lowered in animals of the 1st group down to $4.31 \pm 0.03 \mathrm{~g} / 1$ and down to $4.10 \pm 0.01 \mathrm{~g} / 1(\mathrm{P}<0.001)$ in the $2 \mathrm{nd}$ group. Such change in the level of $\beta_{2}$-globulins is primarily connected with toxic action of cadmium, because our further studies have shown high concentrations of cadmium in kidneys and destructive changes in proximal kidney tubules to suppress reabsorption of $\beta_{2}$-globulins.

The data on the protein spectrum of cow blood testifies that the long-term impact of a qualitatively new factor of the medium (toxic elements) shifts the homeostasis, which through higher regulatory mechanisms of brain (hypothalamus-pituitary systems) activates the systems responsible for adaptation $[1,6]$. The hypothalamus through releasing hormones connects the neural system with endocrine one (neural impulses transform into humoral impulses). This also mobilizes the functional system specifically responsible for adaptation, growth of cellular structures limiting the functional level of cells and organs responsible for the adaptation. At the same time, high concentration of immunosuppressors weakening the immune function usually affects the processes connected with the fission of immunocompetent cells. In out study, it is confirmed by decreased concentration of gamma-2-globulins in cows from both groups down to $8.65 \pm 0.07$ and $8.01 \pm 0.11 \mathrm{~g} / 1(\mathrm{P}<0.01)$, respectively, with average normal indicator of $9.92 \pm 0.056 \mathrm{~g} / \mathrm{l}$. Noteworthily, the synthesis of gamma-2-globulins is a specific function of immunocompetent cells and can reflect the condition of the immunity cellular system. Also, this necessitates the study of cellular immunity indicators $[2,7]$.

The ferments of alanine-aminotransferase (ALT) and aspartate transaminase (AST) that belong to the group of organ-specific or indicating ferments play an important role in establishing the specific ways of protein metabolism. We have established that the activity of aminotransferase of cow blood serum in animals of both groups has pretty the same behavior. In particular, the indicator of activity of glutamic oxaloacetic transaminase with high degree of accuracy is increased in animals in the 1 st group by $86.03 \%$ and amounts to $4650.69 \pm 45.26$ nkat $/ 1 \quad(\mathrm{P}<0.001)$ and by $52.61 \%$ (3815.16 $\pm 29.72 \mathrm{nkat} / \mathrm{l}, \mathrm{P}<0.001)$ in the $2 \mathrm{nd}$ group. It is widely known that AST and ALT exist in two molecular forms: mitochondrial and cytosolic; however, about a third of total activity of GOT is localized in mitochondria, while the larger part of ALT is in cytosol. The result show that abnormal amounts of microelements in environmental objects cause intense growth of activity of the cytoplasmic ferment. The activity of ALT in cows of the 1st group increased 2.27 times and 1.73 times in the 2nd group; the activity of AST increased by 86.03 and $52.61 \%$ respectively in the two groups, which indicates the disturbance of the structure and function of hepatocytes primarily accompanied by liberation into blood of cytosolic ferment (ALT)
Our suggestion on pathologic changes in cows' liver are confirmed by the AST/ALT ratio $(2.49 ; \mathrm{P}<0.01$ and 2.68; $\mathrm{P}<0.001$, respectively). The increase of this indicator testifies the appearance of a pathologic process in liver that promotes increased permeability of cellular membranes.

Thus, the results of biochemical studies of cow blood serum demonstrating the indicators of protein metabolism in the organism and comparison of them with reference data allow suggesting that the complex and sophisticated impact of anthropogenic environment pollution cause pseudoadaptation of animals that temporarily compensates hidden pathologic processes.

Under the conditions of biopathogenic zone characterized by the cows' diet lacking cobalt and zinc and having excessive iron, copper, manganese, nickel, lead and cadmium, the metabolism of cows is disturbed, which affects their productivity. Long-term exposure of cows' organisms to adverse environmental factors lead to reduced content of albumins, beta- and gamma-two-globulins in blood, which witnesses inhibited humoral factor of natural resistance of cows in the first half of gestation length. This causes reduced provision of necessary plastic materials for intensely developing embryos, which mainly inhibits their development and predetermines physiological maldevelopment of calves [9].

Thus, the results of biochemical studies of cow blood serum demonstrating the indicators of protein metabolism in the organism and comparison of them with reference data allow suggesting that the complex and sophisticated impact of anthropogenic environment pollution cause pseudoadaptation of animals that temporarily compensate hidden pathologic processes.

Under the conditions of biopathogenic zone characterized by the cows' diet lacking cobalt and zinc and excess of iron, copper, manganese, nickel, lead and cadmium, the metabolism of cows is disturbed, which affects their productivity. Longterm exposure of cows' organism to adverse environmental factors lead to reduced content of albumins, beta- and gammatwo-globulins in blood, which witnesses inhibited humoral factor of natural resistance of cows in the first half of gestation length. This causes reduced provision of necessary plastic materials for intensely developing embryos, which mainly inhibits their development and predetermines physiological maldevelopment of calves.

The study results have demonstrated the total blood glucose of cows from the 1st and 2nd groups to be lower than the reference values by $13.85 \ldots 15.82 \%$. Such glucose level in the cows' blood $(2.98 \pm 0.09 \ldots 3.05 \pm 0.18 \mu \mathrm{mol} / \mathrm{l})$, from our perspective, has direct connection with the total protein content and character of the blood serum proteinogram. Probably, glucose under such conditions is supplied basically due to gluconeogenesis reactions from aminoacids involved into metabolism, while the gluconeogenesis is supported by the regulatory participation of parasympathetic nervous system, which prevents the appearance of clearly pronounced hypoglycemia.

The alteration of the content of carbohydrate metabolites in the cows' blood are one-directional and confirm our results on 
impaired liver function; however, the intensity of the alteration is different. For example, the concentration of pyroracemic acid-the main metabolite of glycolysis and gluconeogenesis - amounts to $119.17 \pm 9.95 \mu \mathrm{mol} / \mathrm{l}$ for the animals from the 1 st group and $122.01 \pm 8.04 \mu \mathrm{mol} / \mathrm{k}$ for the animals from the 2 nd group, which reliably exceeds the reference values by $21.0 \%$ on average. At the same time, the concentration of lactate - the end-product of glycolysisincreases more significantly $(1.98 \pm 0.03 \mathrm{mmol} / \mathrm{l}$ and $2.22 \pm 0.06$ $\mathrm{mmol} / \mathrm{l}$ for respective groups, $\mathrm{P}<0.001)$ and exceeds the reference values by 62.17 and $82.04 \%$, correspondingly.

The accumulation of lactic acid indicates the deficit of oxygen in cells, which converts carbohydrate metabolism from aerobic into anaerobic one, and the main way of Dglucose transformation becomes anaerobic glycolysis.

The analysis of received data on the content of pyroracemic and lactic acids allows concluding that the organism of cows under the action of adverse environmental factors suffers from energy deficit. This is also testified by the data on lactate/pyruvate ratio. This indicator reliably increases by $36.15 \%(\mathrm{P}<0.001)$ for animals from the 1 st group and by $49.18 \%(\mathrm{P}<0.001)$ for those from the 2 nd group.

The aforementioned data tells about the marks of hypoxic carbohydrate metabolism, despite the balanced diet in terms of protein and glucose content; it also indicates direct participation of heavy metals in hypoxia development. Probably, their high concentrations (primarily, lead content) cause functional and morphological changes in mitochondria and suppress breathing, phosphorylation and transporting, which, evidently, conditions a number of pathologic effects of this metal. In addition, the disturbance of the balance of oxidation phosphorylation and breathing can occur under the lack of cadmium.

An important intermediate glycolysis reaction related with the energy liberation is oxidoreduction between phosphoglyceric aldehyde and pyroracemic acid [5].

In our investigation, the reduced intensity of glycolitic phase of carbohydrate decomposition is confirmed by changed activity of fructose-bisphosphate aldolase. The comparative analysis of the ferment activity in the cows from the test groups with reference values demonstrates its reduction. The activity of fructose-bisphosphate aldolase in the organisms amounts to $91.34 \pm 11.23$ nkat/l $(\mathrm{P}<0.01)$ and $76.64 \pm 14.25$ nkat/1 $(\mathrm{P}<0.001)$, respectively, which is $1.49 \ldots 1.57$ times lower than normal value and indicates functional liver disease.

An important role in metabolism, including energy metabolism in an animal's organism is played by phosphatases or hydrolases of glucose-phosphate monoesters, splitting off phosphoric acid radical with the formation of unbound glucose in the case of phosphorolytic way of liver glycogen sciccion (glycogen mobilization in case of hypoglycaemia). The investigation results show $1.63 \ldots 1.75$ times suppressed activity of alkaline phosphatase. Obviously, the suppression of the alkaline phosphatase activity is promoted by the accumulation of metabolites of abnormal metabolism. Probably, the decrease in the ferment activity is connected with the established intensification of calcium-phosphorous metabolism, which can be explained by enhanced mobilization of these elements from the bones to form tissues and organs of the embryo and preparation of the mammary gland.

All afore described peculiarities of altered carbohydrate metabolism of cows are connected with the fact that during this period the maternal organism suffers deep morphological and physiological changes bound with enhanced metabolic processes in cow organism. They have dual destination: firstly, the mobilization of intraorganic sources of energy supply for maternal organism and forming embryos under hypoxia and predominantly anaerobic oxidation of carbohydrates; secondly, the plastic function to form the structure of organs and tissues of embryos. They also testify the disturbed metabolism in the organism of pregnant cows dwelling in ecologically adverse zones.

The decrease of the main energy source, carbohydrates, in the cows' blood has determined the birth of still-born and maldeveloped calves, and in particularly hard cases, embryotocia and warps. The level of blood glucose is known to be provided by the glycogen in the organism's repository and sugar from food that procure necessary amount of glucose phosphate for glycolysis, Krebs cycle, respiratory chain and oxidative phosphorylation. The toxic elements cause depletion of energy resources, which disturbs the carbohydrate metabolism. This occurs due to suppression of ATPadrenaline dependent resources of glycogenolysis This deteriorates the usage of galactose and other food sugar. Glucose in such conditions is procured through gluconeogenesis reactions that are mainly provided under metabolic regulation by parasympathetic nervous system. Parasympathetic metabolism regulation being a reserve (shunting) way prevents the hypoglycemic condition and is not regulated by the lack of energy of high-energy compounds.

Thus, the inclusion of the shunting into the energy metabolism over a long time gradually decreases the aminoacid reserve of the organism, in so doing suppressing the development rate and final formation of embryos, as well as the gain of live weight of calves after their birth.

Noteworthily, hypoglycaemia and mainly anaerobic oxidation of carbohydrates promote lipolysis. In this connection, the study of lipoid metabolism is of particular interest.

The studied cows demonstrate the tendency to increased total concentration of lipides, which amounts to $6.15 \pm 0.40$ and $6.24 \pm 0.32 \mathrm{~g} / \mathrm{l}$, correspondingly to the groups. However, the differences with the reference values are reliable only for the cows from the 2 nd group and amount to $6.30 \%(\mathrm{P}<0.05)$.

Taking into account that exo- and endogenic lipides with blood and lymph are transported as water-soluble lipideprotein biocomplexes (lipiproteins), we have determined the level of $\beta$-lipoproteins in cows' organisms.

The result show that the decrease in antioxidative capability of blood serum, evidently, is one of the reasons of augmented synthesis of low-density lipoproteins. For instance, the concentration of $\beta$-lipoproteins increased with high reliability for cows dwelling in the zone polluted with toxic 
elements, which is testified by the difference between the reference and absolute values of the studied indicator of 20.13..32.01\%. Moreover, more intense synthesis of $\beta$ lipoproteins is noted for the animals from the 1st group $(8.00 \pm 0.11 \mathrm{~g} / 1$ and $7.28 \pm 0.10 \mathrm{~g} / \mathrm{l} ; \mathrm{P}<0.001$ with normal value of $6.06 \pm 0.07 \mathrm{~g} / 1$ ).

$\beta$ - lipoproteins are the end-product of katabolism of pre- $\beta$ (very low density) lipoproteins and chylomicrons (Chm). Probably, the higher katabolism rate over the rate of chylomicron biosynthesis in intestinal tract and pre- $\beta$ lipoproteins in liver, firstly, lowers their very concentrations, as well as the concentration of high-density lipoproteins (HDL) of albumin zone (as explained above, our studies have established reliable decrease of total concentration of albumin fraction of protein assay), and secondly, indicates changed lipoid metabolism in general. Also, one should note that certain accumulation of $\beta$-lipoproteins in cow blood under disturbed lipoid metabolism occurs due to their comparatively long biological half-life of 3.3 days $[2,6,10]$.

\section{CONCLUSIONS}

The delayed biosynthesis of proteins providing immunobiological resistance of cows' organisms leads to involvement of aminoacids into indirect deamination and transamination, which is confirmed by the behavior of changing activity of transaminases, main ferments providing normal gluconeogenesis through involvement into the process of aminoacid metabolism products and promotes suppression of alkaline phosphatase activity, which also improves energy metabolism. This results in substantial reconfiguration of neurohumoral and immune adaptation regulation mechanisms, accompanied by high energy consumption.

In general, the established changes witness characteristic disturbance of intermediate lipide metabolism in studied cows, which is expressed as lipidemia and hyper- $\beta$-proteinemia.

\section{References}

[1] M.A. Chasovshchikova, O.M. Sheveleva, M.A. Svjazhenina, N.I Tatarkina, A.B. Satkeeva, A.A. Bakharev, "Relationship between the genetic variants of kappa-casein and prolactin and the productivebiological characteristics of cows of the black-motley breed", Journal of Pharmaceutical Sciences and Research, 2017, Vol. 9, No. 7, pp. 10381044

[2] S.D. Batanov, O.S. Starostina, "Blood as regulator of metabolism in organism" (in Russian), Proc. Int. sci. pract. conf. in 3 volumes, Izhevsk State Agricultural Academy, 2018, p. 250-252.

[3] S.D. Batanov, O.S. Starostina, "Realization of productive and reproductive potential of cattle. Prognosis of effective production of beef" (in Russian), Agriculture, vol. 2, No. 74, 2017, pp. 20-27.

[4] V.E. Ulitko, Yu.V. Semyonova, E.V. Savina, L.A. Pykhtina, O.A. Desyatov, "Productivity of hogs on implementation of diet with feed supplement with sorbing and probiotic properties" (in Russian), Zootekhniya, 2018, No. 7, pp. 25-27.

[5] R.V. Nekrasov, M.G. Chabaev, A.A. Zelenchenkova, "Ferment agents for increased metabolism and productivity of growers" (in Russian), Hog rearing, 2018, No. 4, pp. 37-38

[6] M. Chabaev, R. Nekrasov, A. Zelenchenkova, "Fidbest WP will improve the growth of growers. Efficacy of ferment agents in formulated feed" (in Russian), Hog rearing, 2017, No. 4, pp. 33-35.

[7] G.A. Yarmots, A.B. Satkeeva, L.P. Yarmots, "Impact of organic selenium on animal blood indicators" (in Russian), Feeding of agricultural animals and foods production, 2016, No. 7, pp. 49-57.

[8] O.G. Lorets, O.V. Gorelik, S.A. Gritsenko, A.A. Belookov, "Genetic parameters of biochemical composition of milk and blood of dairy cows" (in Russian), Agrarian bulletin of Urals, 2017, vol. 164, No. 10.

[9] O.G. Lorets, O.S. Chechenikhina, O.A. Bykova, A.V. Stepanov, E.S Kazantseva, O.V. Gorelik, "Increased productive live of black pied cows" (in Russian), Yekaterinburg, 2017.

[10] R.R. Fatkullin, A.A. Ovchinnikov, E.M. Ermolova, Y.V. Matrosova Y.V., S.A. Chulichkova, "Antioxidant system and its functioning in animal organisms", International Journal of Engineering and Technology(UAE), 2018, Vol. 7, No. 3.14, pp. 300-304. 